\title{
Turbulence Statistics in a Two-Dimensional Vortex Condensate
}

\author{
Anna Frishman ${ }^{1,2, *}$ and Corentin Herbert ${ }^{3,2, \dagger}$ \\ ${ }^{1}$ Princeton Center for Theoretical Science, Princeton University, Princeton, New Jersey 08544, USA \\ ${ }^{2}$ Department of Physics of Complex Systems, Weizmann Institute of Science, P.O. Box 26, Rehovot 76100, Israel \\ ${ }^{3}$ Univ Lyon, ENS de Lyon, Univ Claude Bernard, CNRS, Laboratoire de Physique, Lyon F-69342, France
}

(Received 9 November 2017; published 18 May 2018)

\begin{abstract}
Disentangling the evolution of a coherent mean-flow and turbulent fluctuations, interacting through the nonlinearity of the Navier-Stokes equations, is a central issue in fluid mechanics. It affects a wide range of flows, such as planetary atmospheres, plasmas, or wall-bounded flows, and hampers turbulence models. We consider the special case of a two-dimensional flow in a periodic box, for which the mean flow, a pair of box-size vortices called "condensate," emerges from turbulence. As was recently shown, a perturbative closure describes correctly the condensate when turbulence is excited at small scales. In this context, we obtain explicit results for the statistics of turbulence, encoded in the Reynolds stress tensor. We demonstrate that the two components of the Reynolds stress, the momentum flux and the turbulent energy, are determined by different mechanisms. It was suggested previously that the momentum flux is fixed by a balance between forcing and mean-flow advection: using unprecedently long numerical simulations, we provide the first direct evidence supporting this prediction. By contrast, combining analytical computations with numerical simulations, we show that the turbulent energy is determined only by mean-flow advection and obtain for the first time a formula describing its profile in the vortex.
\end{abstract}

DOI: 10.1103/PhysRevLett.120.204505

More often than not, turbulence appears hand in hand with a coherent mean flow. At high Reynolds numbers, the interactions between the two are strong, and closed equations describing each component separately cannot be obtained. This is a central problem in fluid mechanics [1], with far-reaching consequences. Indeed, turbulence retroacts on the mean flow, through a term called the "Reynolds stress tensor," which encapsulates the important physical quantities: the turbulent momentum flux and the turbulent energy. For instance, numerical models that do not fully resolve turbulence require a parametrization of the Reynolds stress [2]. This term also drives atmospheric jets such as the jet stream, which governs weather at midlatitudes [3]. It is therefore crucial to better understand the statistics of this object.

While an understanding of the interactions between a mean flow and turbulence remains elusive for general 3D flows, progress can be made for the class of quasi-2D flows, in which turbulence can spontaneously generate a mean flow, rather than feeding on it [4]. This occurs in 2D turbulence [5], on which we focus next, but also in largescale geophysical flows [3]. In such flows, the turbulent energy tends to be transferred towards increasingly larger scales. Eventually, if the large-scale dissipation mechanism is slow enough, energy accumulates at the domain scale and a mean flow emerges, referred to as a condensate [6]. Its structure depends on the domain geometry: in the following, we consider the vortex condensate, which appears in a square box or on a flat torus (as part of a vortex dipole). The condensate is expected to become asymptotically strong compared to turbulence when the large-scale dissipation rate tends to zero, which justifies a perturbative treatment. More precisely, if the mean-flow shear rate is much larger than the rate of nonlinear turbulence-turbulence interactions, then the latter can generically be neglected. This is called the quasilinear approach: it has inspired statistical closures for quasi-2D flows, such as stochastic structural stability theory [7] or, equivalently, cumulant expansion [8]. The idea has also been applied to 3D wall turbulence [9] and investigated numerically in atmospheric dynamics [10]. For a pronounced mean flow, this approach can be justified theoretically using adiabatic reduction [11], but has some success also outside that regime [12]. Actually, if turbulence is excited at asymptotically small scales, the perturbative treatment allows us to analytically derive an explicit formula for the mean-flow and momentum flux profiles, as demonstrated for the vortex condensate $[13,14]$ and discussed for jets $[15,16]$ and on the sphere [4]. Until now, the only part of these predictions that was quantitatively checked against data from direct numerical simulation (DNS) is the profile of the mean flow [13].

In this Letter, we present new results on the statistics of the Reynolds stress in 2D. First, using long time integration, we provide the first numerical evidence supporting the explicit formula for the momentum flux [13,14]. Second, we show that the turbulent energy is determined by a different mechanism: while the momentum flux results from a balance between forcing and shear at small scales, 
TABLE I. Parameters for the DNS runs.

\begin{tabular}{ccccccc}
\hline \hline & $Z$ & $A$ & $B$ & $C$ & $D$ & \multicolumn{1}{c}{$E$} \\
\hline $10^{5} \times \alpha$ & 20 & 11 & 5.5 & 2.5 & 1.25 & 0.625 \\
$10^{3} \times \delta$ & 12.3 & 8.14 & 4.58 & 2.4 & 1.45 & 1.09 \\
\hline \hline
\end{tabular}

the energy is determined solely by advection at large scales. We explain its structure by combining a self-consistent theoretical framework and numerical results, hence describing the full Reynolds tensor. To our knowledge, this is the first time that such explicit formulas are derived either in 2D or 3D turbulence. Furthermore, while the results for the mean flow and momentum flux rely on features specific to 2D turbulence, the mechanism governing turbulent energy may apply more generally.

Framework and numerical methods.-We consider an incompressible flow on a square domain of length $L$ with periodic boundary conditions, with linear friction as the large-scale dissipation mechanism. The governing equations for the velocity field $\mathbf{v}$ are the 2D Navier-Stokes equations

$$
\partial_{t} \mathbf{v}+\mathbf{v} \cdot \nabla \mathbf{v}=-\nabla P-\alpha \mathbf{v}-\nu(-\Delta)^{p / 2} \mathbf{v}+\mathbf{F},
$$

where $P$ is the pressure, $\alpha$ is the friction coefficient, $\nu$ is the hyperviscosity, and $\mathbf{F}$ is a random forcing. We work with an isotropic, white in time forcing acting in a narrow shell in Fourier space centered on wave number $K_{f}$, with $\varepsilon=\langle\mathbf{v} \cdot \mathbf{F}\rangle$ the average energy injection rate [17].

DNS results are obtained by integrating (1) using the GHOST pseudospectral code, at resolution $512^{2}$ and $1024^{2}$, with parameters $L=2 \pi, K_{f}=L / \ell_{f}=100, p=16$, and $\nu=5 \times 10^{-35}$. We carried out runs covering almost 2 orders of magnitude in the friction coefficient $\alpha$ (see Table I). In agreement with previous studies, the flow reaches a condensate steady state, taking the form of a vortex dipole (see Fig. 1). The vortices drift slowly across the box, with fast turbulent fluctuations superimposed onto them. We carry out a Reynolds decomposition in polar coordinates centered on one of the vortices: the mean flow $\langle\mathbf{v}\rangle=U \mathbf{e}_{\phi}$ is purely azimuthal, while the fluctuations read $\tilde{\mathbf{v}}=v \mathbf{e}_{r}+u \mathbf{e}_{\phi}$. Angular brackets $\langle\cdot\rangle$ denote a time average [21]. The dimensionless parameter $\delta=\alpha L^{2 / 3} / \varepsilon^{1 / 3}$, introduced as the ratio between the condensate spin-down time $\alpha^{-1}$ and the eddy turnover time $L^{2 / 3} \varepsilon^{-1 / 3}$, measures the timescale separation.

As shown previously $[13,14,16]$, within the quasilinear approximation— justified for $\delta \ll 1$-and once $K_{f} \gg 1$, so that the mean flow can be approximated locally by a uniform shear, it is possible to derive an explicit formula for the mean flow $U$ and momentum flux $\langle u v\rangle$

$$
U=\sqrt{3 \varepsilon / \alpha}, \quad\langle u v\rangle=-r \sqrt{\alpha \varepsilon / 3}
$$

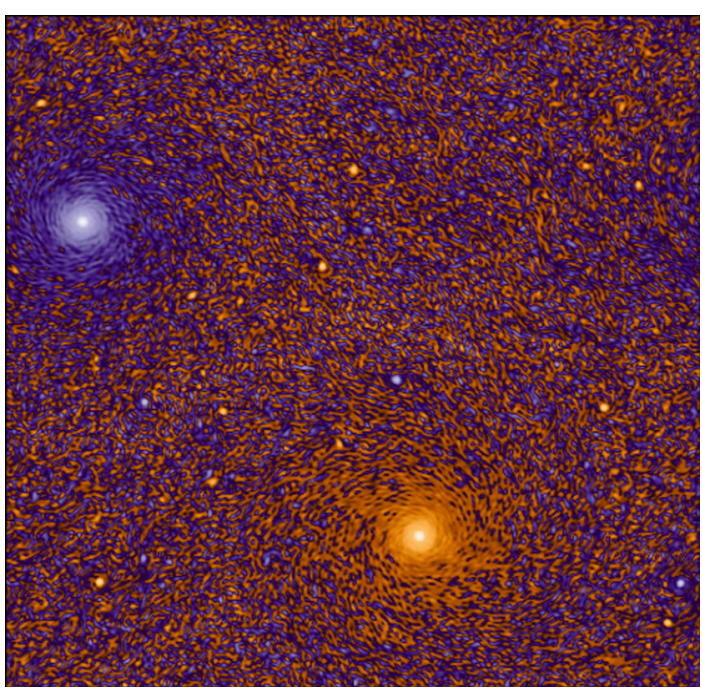

FIG. 1. Snapshot of the vorticity field $\left(1024^{2}\right)$ in the stationary state of the DNS; the vortex dipole forming the condensate is clearly visible.

This profile is expected to hold in the range $\ell_{f} \ll r \ll R_{u}$, where $R_{u}=\delta^{-1 / 2} K_{f}^{-2 / 3} L$ measures the radius where the rate of nonlinear interactions is comparable to the meanflow shear rate. At larger radii, the quasilinear approximation is expected to break, while for $r \ll \ell_{f}$, the uniform shear approximation for the mean flow is no longer applicable. Of course, once $R_{u} \gtrsim L$, the range of validity should be set by the boundary-be it a wall or a second vortex. The mean-flow profile in our simulations (shown in the Supplemental Material [17]) is compatible with the theoretical prediction in a region that expands with decreasing $\delta$. This is consistent with earlier numerical results [13], over a wider range of friction coefficient $\alpha$. In the figures, we represent the range $\ell_{f} \ll r \ll R_{u}$ by a shaded area and the empirical range of validity by vertical dashed lines. Focusing on this region, we now discuss the three terms of the average Reynolds stress tensor: $\langle u v\rangle$, $\left\langle u^{2}\right\rangle$, and $\left\langle v^{2}\right\rangle$.

The average momentum flux profile.-Until now, unlike the mean flow, no experimental or numerical evidence has been given for the average momentum flux (2). The difficulty is that it is a small quantity $\left[\langle u v\rangle / U^{2}=O\left(\delta^{3 / 2}\right)\right]$ and it is not sign definite: the average value results from cancellations of strongly fluctuating contributions. Leveraging the hybrid parallelization of GHOST [22], we were able to integrate our runs over extremely long times (about 320000 turnover times) and accumulate enough statistics to observe partial convergence of the average momentum flux $\langle u v\rangle$, shown in Fig. 2. Although convergence is restricted to a subregion of the region of interest that does not match the empirical range defined above, we find that the momentum flux is consistent with the prediction (2): the numerical data unambiguously confirm the negative sign and the scaling with $\alpha$ and are compatible 


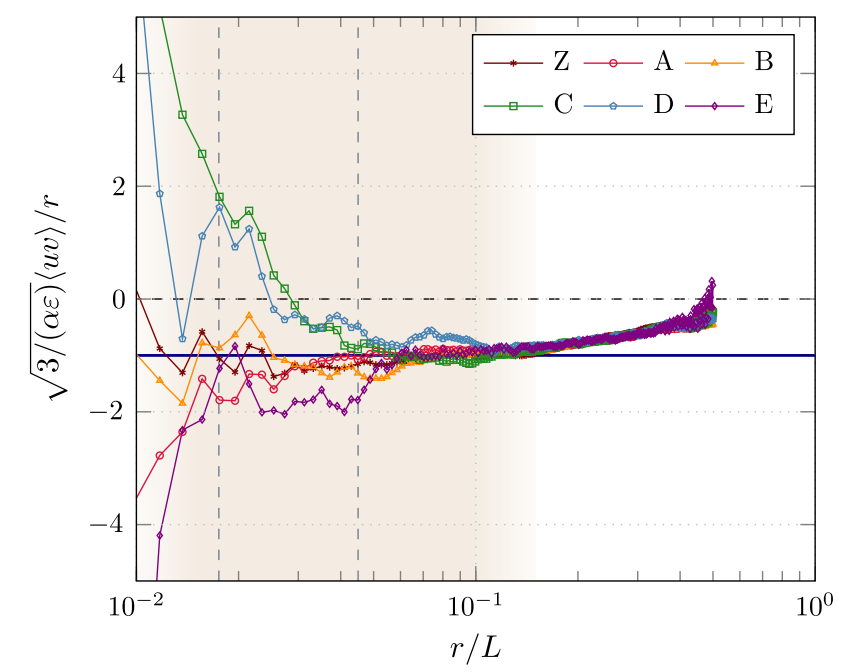

FIG. 2. Rescaled profile of average momentum flux $\langle u v\rangle$ from DNS. The horizontal blue line corresponds to the theoretical prediction. Vertical dashed lines denote the range where the mean-flow profile (2) is observed to hold.

with the theoretical prediction at the outer edge of the region, $r / L \lesssim 10^{-1}$ (smaller radii have less statistics).

The average turbulent energy profile.-Symmetry considerations imply that the magnitude of the diagonal terms of the Reynolds tensor, $\left\langle u^{2}\right\rangle$ and $\left\langle v^{2}\right\rangle$, as well as the mechanism that dictates them, are different than those for the momentum flux $[13,14]$. We therefore take a different approach than [23], where the turbulent energy is computed within the same framework used for the momentum flux. Instead, we will show that $\left\langle u^{2}\right\rangle$ and $\left\langle v^{2}\right\rangle$ are determined by the zero modes of the advection equation for two-point correlation functions. Indeed, in the quasilinear framework, fluctuations are linearly advected by the mean flow. Because of incompressibility, the dynamics is characterized by a single field: vorticity, for instance, obeys the equation $\left(\partial_{t}+\mathcal{D}\right) \omega+L_{U} \omega=f_{\omega}$, with $\mathcal{D}=\alpha+\nu(-\Delta)^{p / 2}$, and $L_{U} \omega=(U / r) \partial_{\phi} \omega+\Omega^{\prime} v$. From here, a closed equation for two-point correlation functions directly follows; for $\Phi=\left\langle\omega_{1} \omega_{2}\right\rangle$, with $C=\left\langle f_{\omega 1} f_{\omega 2}\right\rangle$, the steady-state advection equation reads $\left[L_{U}^{(1)}+L_{U}^{(2) \dagger}+\mathcal{D}_{1}+\mathcal{D}_{2}^{\dagger}\right] \Phi=2 C$. In general, the operator $L_{U}$ is nonlocal and this is an integrodifferential equation (mixed $\left\langle\omega_{1} v_{2}\right\rangle$ terms appear). The mean-flow profile (2), however, holds only in part of the domain. Furthermore, we are eventually interested in velocity statistics. Therefore, we transform this equation into a partial differential equation for the radial velocity correlation function, using incompressibility: $\partial_{\phi_{1}} \partial_{\phi_{2}} \Phi=$ $\Delta_{1} \Delta_{2} r_{1} r_{2}\left\langle v_{1} v_{2}\right\rangle$. We now claim that $\left\langle v_{1} v_{2}\right\rangle$ is dominated by zero modes of the resulting advection operator. This entails two approximations. First, we neglect the contribution from dissipation. This is justifiable in the region $r_{1}$, $r_{2} \ll R_{u}$, where fluctuations are weak compared to the mean flow. Second, we neglect the injection by the forcing, which is justified as long as $\left|r_{1}-r_{2}\right| \gg \ell_{f}$. In fact, we eventually need to take the limit $r_{1} \rightarrow r_{2}$, passing through $\ell_{f}$, to compute the energy $\left\langle u^{2}\right\rangle$ and $\left\langle v^{2}\right\rangle$. However, for $K_{f} \gg 1$, we expect to recover the correct result in this limit, since velocity correlation functions should be continuousthe energy being mainly determined at scales larger than the forcing scale. We shall solve explicitly the resulting homogeneous advection equation characterizing the zero modes, with the mean-flow profile (2). It reads

$$
\left[\mathcal{L}_{2} r_{2}\left(2 \partial_{r_{1}} r_{1}+\mathcal{L}_{1}\right)-\mathcal{L}_{1} r_{1}\left(2 \partial_{r_{2}} r_{2}+\mathcal{L}_{2}\right)\right] \partial_{\phi_{1}}\left\langle v_{1} v_{2}\right\rangle=0
$$

with the notation $\mathcal{L}_{i}=r_{i}^{2} \Delta_{i}$, using isotropy $\left(\partial_{\phi_{1}}=-\partial_{\phi_{2}}\right)$.

Given the form of the advection operator, it is natural to decompose the fluctuations into angular harmonics: $v(r, \phi)=\sum_{m=-\infty}^{\infty} \hat{v}_{m}(r) e^{i m \phi}$ (and similar for $u$ ). The resulting equation for $\left\langle\hat{v}_{m}\left(r_{1}\right) \hat{v}_{m}^{*}\left(r_{2}\right)\right\rangle$ is scale invariant and independent of the value of the mean flow $U$. This prompts the ansatz $\left\langle\hat{v}_{m}\left(r_{1}\right) \hat{v}_{m}^{*}\left(r_{2}\right)\right\rangle=r_{1}^{\lambda} f_{m}\left(r_{2} / r_{1}\right)$, which allows us to convert our partial differential equation into an ordinary differential equation in the variable $R=r_{2} / r_{1}$. It can be written compactly as the hypergeometric equation [24]:

$\prod_{i=1}^{4}\left(R \frac{d}{d R}-\gamma_{i}\right) f_{m}(R)=R \prod_{i=1}^{4}\left(R \frac{d}{d R}+\alpha_{i}\right) f_{m}(R)$,

with the parameters $\left(\gamma_{1}, \gamma_{2}, \gamma_{3}, \gamma_{4}\right)=(\bar{\lambda}-m, \bar{\lambda}+m,-1+$ $\left.\sqrt{m^{2}-1},-1-\sqrt{m^{2}-1}\right), \quad\left(\alpha_{1}, \alpha_{2}, \alpha_{3}, \alpha_{4}\right)=(1-m, 1+m$, $\left.-\bar{\lambda}+\sqrt{m^{2}-1},-\bar{\lambda}-\sqrt{m^{2}-1}\right)$, and $\bar{\lambda}=\lambda+1$. This equation has four families of solutions, described in detail in the Supplemental Material [17], each parametrized by $m$ and $\lambda$. We now turn to the numerical simulations to identify which solutions derived in our theoretical framework (i.e., which parameters $\lambda$ ) contribute to the turbulent energy profile.

Decomposing the DNS data into harmonics, we see that turbulent energy in the region of interest is strongly dominated (about $90 \%$ ) by the $m=1$ modes (see the dashed curve in Figs. 3 and 4 and also the Supplemental Material [17]). Figure 3 also suggests that, in the universal region, $\left\langle\left|\hat{u}_{1}\right|^{2}\right\rangle \approx \Im\left\langle\hat{u}_{1} \hat{v}_{1}^{*}\right\rangle \approx$ const and $\left\langle\left|\hat{v}_{1}\right|^{2}\right\rangle \approx$ const $+r^{\beta}$ with some $\beta<0$. Comparing this form to the possible solutions to (4), we find that it corresponds to a unique superposition of two solutions, one with $\lambda=0$, which has $\left\langle\left|\hat{u}_{1}\right|^{2}\right\rangle=\left\langle\left|\hat{v}_{1}\right|^{2}\right\rangle$, and the second with $\lambda=\beta=-2$ [17]. Indeed, a very good match to such a power law can be seen in Fig. 4, once $\left\langle\left|\hat{u}_{1}\right|^{2}\right\rangle$ is subtracted from $\left\langle\left|\hat{v}_{1}\right|^{2}\right\rangle$. In its current form, our theory does not determine the scaling of the correlation functions with $\delta$. We again turn to the DNS results and find that $\left\langle\left|\hat{u}_{1}\right|^{2}\right\rangle$ and $\Im\left\langle\hat{u}_{1} \hat{v}_{1}^{*}\right\rangle$ collapse for the different runs when rescaled by $\delta^{-1 / 3}$, while the $r^{-2}$ part of $\left\langle\left|\hat{v}_{1}\right|^{2}\right\rangle$ scales like $\delta^{-1}$. However, we also have $U^{2} \propto \delta^{-1}$ 


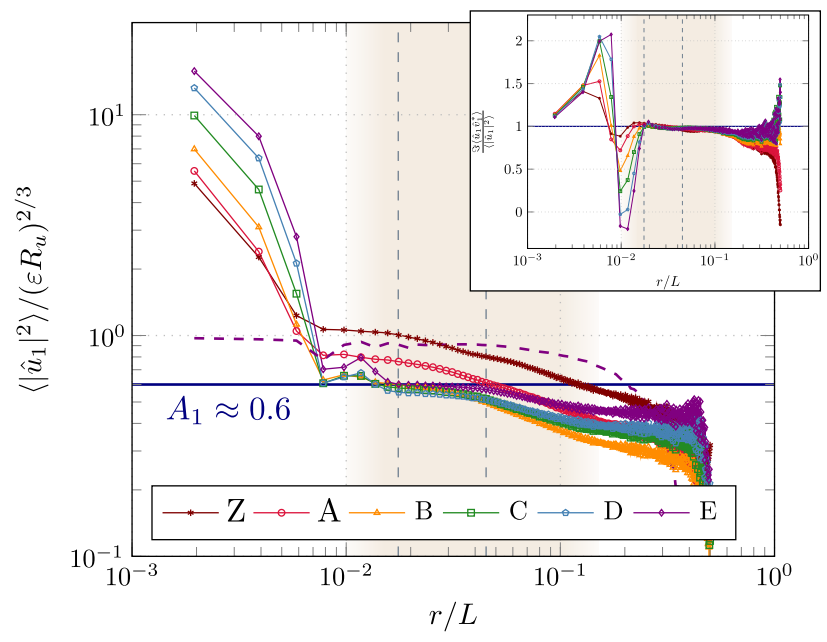

FIG. 3. Profile of the first harmonic $\left\langle\left|\hat{u}_{1}\right|^{2}\right\rangle$ for all the runs, rescaled according to (5). The dashed purple line corresponds to the same quantity, normalized by the full field $\left\langle u^{2}\right\rangle / 2$ for run $E$. (Inset) The ratio $\mathfrak{s}\left\langle\hat{u}_{1} \hat{v}_{1}^{*}\right\rangle /\left\langle\left|\hat{u}_{1}\right|^{2}\right\rangle$.

and we have relied throughout on the ratio $\left\langle\left|\hat{v}_{1}\right|^{2}\right\rangle / U^{2}$ being small. Thus, we expect this term to be suppressed by some power of $K_{f}^{-1}$. We finally obtain

$$
\begin{aligned}
& \left\langle\left|\hat{v}_{1}\right|^{2}\right\rangle=\left(\varepsilon R_{u}\right)^{2 / 3}\left[A_{1}+A_{2}\left(\frac{R_{u}}{L}\right)^{4 / 3}\left(\frac{\ell_{f}}{r}\right)^{2}\right], \\
& \left\langle\left|\hat{v}_{1}\right|^{2}\right\rangle=A_{1}\left(\varepsilon R_{u}\right)^{2 / 3}, \quad\left\langle\hat{u}_{1} \hat{v}_{1}^{*}\right\rangle=i A_{1}\left(\varepsilon R_{u}\right)^{2 / 3},
\end{aligned}
$$

where the dependence on $K_{f}$ is a plausible guess leading to order one numerical coefficients $A_{1}$ and $A_{2}$. In this case, we have at most $\left\langle\left|\hat{v}_{1}\right|^{2}\right\rangle / U^{2} \sim K_{f}^{-4 / 3}$, which is obtained at $r=\ell_{f}$. Equation (5) is our main quantitative result, since it provides a formula for the turbulent energy $\left\langle u^{2}\right\rangle$ and $\left\langle v^{2}\right\rangle$.

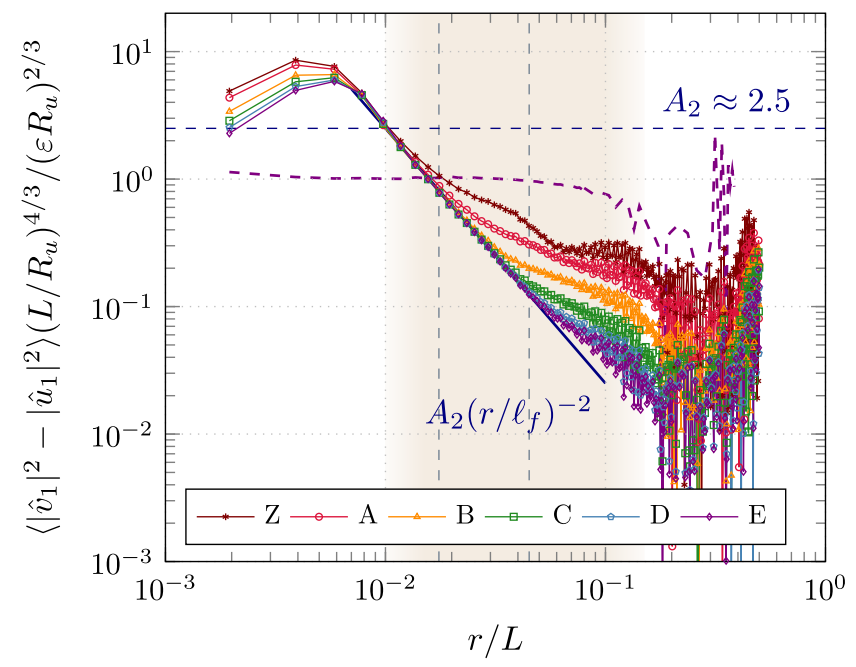

FIG. 4. Rescaled profile of the power law part for the first harmonic $\left\langle\left|\hat{v}_{1}\right|^{2}-\left|\hat{u}_{1}\right|^{2}\right\rangle$, for all the runs. The dashed purple line corresponds to the same quantity, normalized by $\left\langle v^{2}-u^{2}\right\rangle / 2$ for run $E$.
The agreement with these formulas observed in Figs. 3 and 4 improves with decreasing $\delta$. The constant part of the $m=1$ mode is probably due to uniform shifts of the vortex, induced by turbulent fluctuations. A detailed study of such mechanisms is left for future work.

The mode $m=1$, which dominates the energy, is the lowest mode determined by mean-flow advection. Indeed, as a consequence of the isotropy of the mean flow, it does not interact with the $m=0$ harmonic. Note also that the zeroth harmonic of the radial velocity $v$ identically vanishes due to incompressibility. Formulas for higher-order harmonics, however, can be deduced from our formalism. Let us demonstrate that with $m=2$. The DNS results indicate that $\left\langle\hat{u}_{2} \hat{v}_{2}^{*}\right\rangle$ is constant in the universal region. In addition, since $\lambda=-2$ contributes to $m=1$, we check if it contributes to $m=2$ as well. This leads to a superposition of three solutions: the first has $\lambda=0$ and is of the form $f_{2}^{(1)}(R)=R^{\sqrt{3}-1} g(R)$, for $R \leq 1$, where $g(R)$ is a quadratic polynomial. The two other solutions have $\lambda=-2$, and they come in a particular combination, which reads $f_{2}^{(2)}(R)=$ $R^{\sqrt{3}-1}-(\sqrt{3} / 2) R$ for $R<1$ [17]. Like for $m=1$, we use the DNS data to identify the scaling with $\delta$ and deduce the coefficients in the combination of solutions. This gives

$$
\begin{aligned}
\left\langle\left|\hat{v}_{2}\right|^{2}\right\rangle & =\left(\varepsilon \ell_{f}\right)^{2 / 3}\left[B_{1}-B_{2}\left(\frac{R_{u}}{L}\right)^{4 / 3}\left(\frac{\ell_{f}}{r}\right)^{2}\right], \\
\left\langle\left|\hat{u}_{2}\right|^{2}\right\rangle & =\left(\varepsilon \ell_{f}\right)^{2 / 3}\left[\frac{19}{28} B_{1}-\frac{\sqrt{3}}{2} B_{2}\left(\frac{R_{u}}{L}\right)^{4 / 3}\left(\frac{\ell_{f}}{r}\right)^{2}\right], \\
\left\langle\hat{u}_{2} \hat{v}_{2}^{*}\right\rangle & =i \frac{B_{1}}{2}\left(\varepsilon \ell_{f}\right)^{2 / 3} .
\end{aligned}
$$

Figure 5 shows that DNS data seems compatible with (6), except close to the inner boundary of the region, which

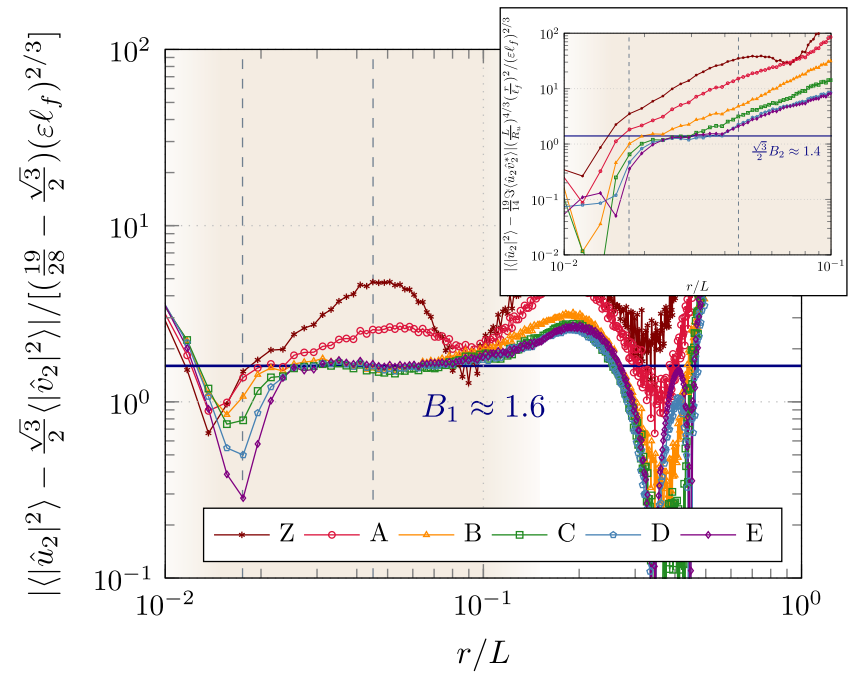

FIG. 5. Combination of the second harmonics demonstrating the constant and $r^{-2}$ (inset) contributions to the profiles (6). 
could either come from the contribution of another solution or a forcing effect.

We finally note that an analog of (3) was used in a previous attempt [25] to determine the momentum flux and mean flow. There, the hierarchy was closed at the level of cubic terms. As was later realized [13,14], this produces a zero momentum flux at any order, because the equations are invariant under the transformation $\phi \rightarrow-\phi, t \rightarrow-t$, while the momentum flux breaks this symmetry. The same happens with the zero modes discussed here: because the coefficients in (4) are real, so are the solutions $\left\langle\hat{v}_{m}\left(r_{1}\right) \hat{v}_{m}^{*}\left(r_{2}\right)\right\rangle$, resulting in $\Re\left[\left\langle\hat{u}_{m} \hat{v}_{m}^{*}\right\rangle\right]=0$.

Conclusion.-In this Letter, we have explored the turbulent fluctuations statistics in a $2 \mathrm{D}$ vortex mean flow sustained by turbulence. We demonstrated that the turbulent energy and momentum flux are governed by different mechanisms, dictated by symmetry. Through a combination of DNS and first-principles theoretical analysis, we showed, for the first time, that the turbulent energy profile (5) - and, more generally, two-point correlation functions - is determined by zero modes of the mean-flow advection equation. The contribution of these zero modes to the momentum flux vanishes, which explains why it is determined at next order by a balance between forcing and advection. We provided the first evidence supporting the resulting profile. A consequence is that the turbulent energy and momentum flux scale differently with the small parameter $\delta$, at variance with the assumption used to justify the quasilinear approach in a kinetic theory framework [11]. Moreover, while the limit $K_{f} \gg 1$ was discussed before [15], our results point to its crucial role in suppressing the fluctuations, which was not considered. We have relied upon DNS results to identify which theoretical solutions are realized and their scaling with $\delta$. This leaves open the question of their selection mechanism. In particular, it would be interesting to check if, in a box with solid boundaries, such as found in experiments, the turbulent energy profile would be the same or if, instead, other solutions would be selected. Our results also represent an important first step for understanding turbulence statistics in more complex flows, such as geophysical jets, plasmas, and ultimately, long-standing problems such as turbulent boundary layers.

The authors would like to thank two anonymous referees for useful comments which helped improve the manuscript. We are grateful to G. Falkovich for the many discussions we had at the initial stages of this work. Computer time was provided by the Weizmann Institute of Science on the Weizmann Exascale Cluster (WEXAC). C. H. would like to thank V. Golodnitsky for technical assistance with the cluster. A. F. is supported by a Princeton Center for Theoretical Science Fellowship.

Authors are listed in alphabetical order and contributed equally to this work. *frishman@princeton.edu

'corentin.herbert@ens-lyon.fr

[1] H. Tennekes and J. L. Lumley, A First Course in Turbulence (MIT Press, Cambridge, MA, 1972); A. A. Townsend, The Structure of Turbulent Shear Flow (Cambridge University Press, Cambridge, England, 1976); S. B. Pope, Turbulent Flows (Cambridge University Press, Cambridge, England, 2000).

[2] P. R. Spalart, Int. J. Heat Fluid Flow 21, 252 (2000); M. Lesieur, O. Métais, and P. Comte, Large-Eddy Simulations of Turbulence (Cambridge University Press, Cambridge, England, 2005).

[3] G. K. Vallis, Atmospheric and Oceanic Fluid Dynamics: Fundamentals and Large-scale Circulation (Cambridge University Press, Cambridge, England, 2017), 2nd ed.; J. Pedlosky, Geophysical Fluid Dynamics (Springer, Berlin, 1987); R. Salmon, Lectures on Geophysical Fluid Dynamics (Oxford University Press, New York, 1998).

[4] G. Falkovich, Proc. R. Soc. A 472, 20160287 (2016).

[5] R. H. Kraichnan and D. C. Montgomery, Rep. Prog. Phys. 43, 547 (1980); G. Boffetta and R. E. Ecke, Annu. Rev. Fluid Mech. 44, 427 (2012).

[6] J. Sommeria, J. Fluid Mech. 170, 139 (1986); L. M. Smith and V. Yakhot, Phys. Rev. Lett. 71, 352 (1993); J. Fluid Mech. 274, 115 (1994); M. Chertkov, C. Connaughton, I. Kolokolov, and V. Lebedev, Phys. Rev. Lett. 99, 084501 (2007); H. Xia, M. Shats, and G. Falkovich, Phys. Fluids 21, 125101 (2009).

[7] B. F. Farrell and P. J. Ioannou, J. Atmos. Sci. 60, 2101 (2003); 64, 3652 (2007); K. Srinivasan and W. R. Young, J. Atmos. Sci. 69, 1633 (2012); N. C. Constantinou, B. F. Farrell, and P. J. Ioannou, J. Atmos. Sci. 73, 2229 (2016).

[8] S. M. Tobias and J. B. Marston, Phys. Rev. Lett. 110, 104502 (2013); J. B. Marston, G. P. Chini, and S. M. Tobias, Phys. Rev. Lett. 116, 214501 (2016); F. Ait-Chaalal, T. Schneider, B. Meyer, and J. B. Marston, New J. Phys. 18, 025019 (2016).

[9] B. F. Farrell, D. F. Gayme, and P. J. Ioannou, Phil. Trans. R. Soc. A 375, 20160081 (2017).

[10] T. Schneider and C. C. Walker, J. Atmos. Sci. 63, 1569 (2006); P. A. O'Gorman and T. Schneider, Geophys. Res. Lett. 34, L22801 (2007).

[11] F. Bouchet, C. Nardini, and T. Tangarife, J. Stat. Phys. 153, 572 (2013).

[12] N. C. Constantinou, B. F. Farrell, and P. J. Ioannou, J. Atmos. Sci. 71, 1818 (2014).

[13] J. Laurie, G. Boffetta, G. Falkovich, I. Kolokolov, and V. Lebedev, Phys. Rev. Lett. 113, 254503 (2014).

[14] I. V. Kolokolov and V. V. Lebedev, Phys. Rev. E 93, 033104 (2016).

[15] E. Woillez and F. Bouchet, Europhys. Lett. 118, 54002 (2017).

[16] A. Frishman, Phys. Fluids 29, 125102 (2017).

[17] See Supplemental Material at http://link.aps.org/ supplemental/10.1103/PhysRevLett.120.204505 for more details about the numerical simulations, including Refs. [18-20] and the analytical derivation of the zero modes. 
[18] G. Boffetta, J. Fluid Mech. 589, 253 (2007); J. A. Domaradzki and R.S. Rogallo, Phys. Fluids A 2, 413 (1990); P. K. Yeung and J. G. Brasseur, Phys. Fluids A 3, 884 (1991); P. Moin and K. Mahesh, Annu. Rev. Fluid Mech. 30, 539 (1998).

[19] B. Legras, P. Santangelo, and R. Benzi, Europhys. Lett. 5, 37 (1988); V. Borue and S. A. Orszag, Europhys. Lett. 29, 687 (1995); A. G. Lamorgese, D. A. Caughey, and S. B. Pope, Phys. Fluids 17, 015106 (2005).

[20] P. B. Rhines, J. Fluid Mech. 69, 417 (1975); H. J. H. Clercx and G. J. F. Van Heijst, Appl. Mech. Rev. 62, 020802 (2009).
[21] The averaging is performed by aligning frames with respect to the vortex center, see further discussion in [17].

[22] P. D. Mininni, D. Rosenberg, R. Reddy, and A. Pouquet, Parallel Comput. 37, 316 (2011).

[23] I. V. Kolokolov and V. V. Lebedev, J. Fluid Mech. 809, R2 (2016).

[24] N. E. Norlund, Acta Math. 94, 289 (1955); A. M. Mathai and R. K. Saxena, Generalized Hypergeometric Functions with Applications in Statistics and Physical Sciences (Springer, New York, 1973).

[25] M. Chertkov, I. Kolokolov, and V. Lebedev, Phys. Rev. E 81, 015302 (2010). 